Vittalle - Revista de Ciências da Saúde v. 32, n. 2 (2020) 17-26

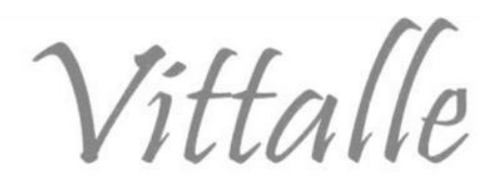

\title{
Caracterização dos casos de malformações congênitas em um município do centro-oeste do Brasil
}

\author{
Rita de Souza Claudino ${ }^{\mathrm{a}^{*}}$, Rafael Henrique da Silva ${ }^{\mathrm{b}}$

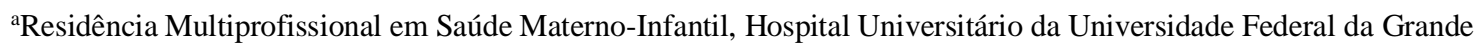 \\ Dourados, Dourados, MS, Brasil

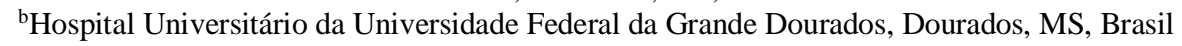

\section{Histórico do Artigo \\ Recebido em: \\ $30 / 11 / 2019$ \\ Aceito em: \\ 20/07/2020}

\section{Palavras-chave:}

Malformações congênitas; sistemas de informação; epidemiologia

\section{Keywords:}

Congenital malformations; information systems; epidemiology

\begin{abstract}
RESUMO
A malformação congênita envolve alterações durante a formação do feto, tanto em um órgão ou sistema, no qual determina uma anomalia morfológica estrutural ou funcional. Objetivou-se caracterizar as principais malformações que acometem recém-nascidos em Dourados, Mato Grosso do Sul e Brasil. Posteriormente, comparar entre os casos levantados, principalmente em relação a incidência, localização, tipo de malformação, idade e escolaridade das mães. Tratou-se de um estudo epidemiológico transversal, quantitativo e descritivo. As fontes de dados foram a partir da Declaração de Nascidos Vivos nas esferas municipal, estadual e nacional no período de 2008 a 2016, através do Sistema de Informação sobre Nascidos Vivos, disponível no site do Departamento de Informática do Sistema Único de Saúde. O número de casos de malformações notificados em Dourados teve um aumento ao longo dos anos totalizando 340, a nível estadual 2.251 e no âmbito nacional 202.410. As malformações predominantes foram aparelho osteomuscular, pé torto congênito, sistema nervoso e fenda labial e palatina. Um comparativo realizado entre os níveis estadual e nacional, observa-se que os casos levantados de MC são análogos. No estado de Mato Grosso do Sul, houve um aumento nos casos notificados, apesar de as causas ainda não serem totalmente conhecidas devido a sua etiologia multifatorial. A média de notificações no estado de Mato Grosso do Sul está acima da nacional. As malformações estão aumentando e para que haja uma redução é necessário realizar educação em saúde com mulheres, principalmente as que estão em período fértil.
\end{abstract}

Characterization of cases of congenital malformation in a city of midwest Brazil

\section{ABSTRACT}

The congenital malformation involves changes during fetal formation, either in an organ or system, in which it determines a structural or functional morphological anomaly. The objective was to characterize the main malformations affecting newborns in Dourados, Mato Grosso do Sul and Brazil. Subsequently compare between the cases raised, especially regarding the incidence, location, type of malformation, age and education of mothers. This was a cross-sectional, quantitative and descriptive epidemiological study. Data were collected from the Live Birth Declaration at the municipal, state and national levels from 2008 to 2016 through the Live Birth Information System, available on the website of the Department of Informatics of the Unified Health System. The number of malformations reported in Dourados has increased over the years totaling 340, at state level 2.251 and nationwide 202.410. The predominant malformations were musculoskeletal system, congenital clubfoot, nervous system and cleft palate. A comparison between the state and national levels shows that the cases of CM are similar. In the state of Mato Grosso do Sul, there was an increase in reported cases, although the causes are not yet fully known due to their multifactorial etiology. The average of notifications in the state of Mato Grosso do Sul is above the national. That malformations are increasing and in order to be reduced, it is necessary to carry out health education with women, especially those who are in fertile period.

\section{Introdução}

As anomalias congênitas são definidas como malformações funcionais ou estruturais durante o desenvolvimento embrionário, decorrente de fatores socioeconômicos,

\footnotetext{
*Autor correspondente: ritadesouzaclaudino@gmail.com (Claudino R.S.)
} 
genéticos, processos infecciosos, fatores nutricionais, materno e ambientais, visto que representa uma das principais causas de mortalidade infantil (1).

O número de casos notificados a partir do Sistema de Informações de Nascidos Vivos (SINASC) encontrados no Departamento de Informática do Sistema Único de Saúde (DATASUS), no ano de 2017 foram de 25.214 casos distribuídos de acordo com cada região. Região Sudeste com 12.201 casos, seguido pela Região Nordeste 6.424 casos, Região Sul 3.250 casos, Região Norte 1.790 e por fim Região Centro-Oeste com 1.549 casos registrados de malformações congênitas (2).

A macrorregião de Dourados possui como relevante alicerce econômico a agropecuária. Assim sendo, o uso de fertilizantes e agrotóxicos para alcance de uma boa produtividade pode trazer sérios problemas ambientais. A aplicação de agrotóxicos se dá através de tratores e até mesmo aviões agrícolas pelo método de pulverização, o que favorece o acometimento da lavoura, dos trabalhadores e vizinhanças que estejam próximos (3).

O uso de agrotóxicos traz uma série de problemas para a saúde, pois ao serem utilizados os mesmos espalham-se pelo meio ambiente, contaminando água, solo e alimentos. Com isso, vários agrotóxicos podem afetar diretamente o ser humano como por exemplo durante o desenvolvimento fetal, levando aos casos de malformações congênitas (4).

Certos grupos populacionais estão vulneráveis a contaminação por agrotóxicos, a exemplo de famílias de agricultores, famílias que residem próximo a locais com grande consumo de agrotóxicos ou até mesmo moradores urbanos, devido a contaminação dos alimentos e água. Dentro os grupos, estudos sugerem que mulheres com exposição aos agrotóxicos durante a fase gestacional apresentam forte relação com malformações congênitas (3).

Trata-se de um estudo epidemiológico do tipo transversal, quantitativo e de caráter descritivo. Os dados foram coletados a partir de Declaração de Nascidos Vivos em nível municipal, estadual e nacional no período de 2008 à 2016, disponibilizados pelo Ministério da Saúde, no SINASC, através do site do DATASUS, programa de livre acesso responsável por contabilizar e classificar informações sobre saúde.

A relevância do trabalho ocorre em virtude da necessidade de identificar quais as malformações que predominam na cidade de Dourados, e correlacionar os níveis municipal, estadual e nacional, esperando assim determinar as características dos casos de malformação congênitas na região.

$\mathrm{O}$ estudo contribui com informações relevantes sobre a correlação entre as MC e fatores ambientais, socioeconômicos possibilitando realizar uma avaliação epidemiológica a fim de identificar as malformações congênitas (MC) de maior incidência e compará-las em esferas superiores, no intuito de observar se o aumento tem caráter regional ou se acompanha as médias estadual e federal.

Desta forma, o presente estudo visou realizar um levantamento das principais malformações que acometem os recém-nascidos na cidade de Dourados, bem como comparar com os números do estado do Mato Grosso do Sul e do Brasil. Posteriormente, objetiva traçar um comparativo entre os casos levantados, correlacionando a incidência, a localização, tipo de malformação, idade das mães e sua escolaridade.

\section{Método}

Trata-se de um estudo epidemiológico do tipo transversal, quantitativo e de caráter descritivo. Os dados foram coletados a partir de Declaração de Nascidos Vivos nos 
níveis municipal, estadual e nacional no período de 2008 à 2016, disponibilizados pelo Ministério da Saúde, no SINASC, acessível no site do DATASUS, sendo este o programa responsável por contabilizar e classificar todos os óbitos ocorridos no país e seu acesso é livre.

Os dados foram apresentados em forma de tabelas, traçando um perfil de malformações que acometem os recém-nascidos e depois correlacionar com as informações sociodemográficas e obstétricas: As questões sociodemográficas, são idade, raça/cor e escolaridade. Os dados obstétricos são idade gestacional e número de consultas. Para análise das informações coletadas, foi utilizado o programa Microsoft Office Excel 2016.

Em relação aos aspectos éticos, os referidos dados estão disponíveis em uma base de dados públicos, justificando a desobrigação de submissão deste projeto ao Comitê de Ética e Pesquisa, conforme a resolução 466/2012 do Conselho Nacional de Saúde, já que nenhum pesquisador ou CEP será identificado. $O$ trabalho foi submetido a Comissão de Avaliação em Pesquisa e Extensão (CAPE) do Hospital Universitário (HU-UFGD/EBSERH) e posteriormente aprovado pelo Parecer 82.2019.

\section{Resultados}

O número de casos de malformações notificados na cidade de Dourados-MS, no período de nove anos (2008 a 2016), foram de 340 casos, sendo que houve 40.372 nascidos vivos, segundo DATASUS. Assim, foram levantadas o número de casos e na tabela seguinte as principais malformações que acometem os recém-nascidos, obtendose os seguintes resultados conforme descritos nas Tabelas 1 e 2.

Tabela 1 - Número de casos referente aos anos de 2008 a 2016 no município de Dourados-MS

\begin{tabular}{lcc}
\hline Casos de malformações & $\mathbf{N}^{\mathbf{0}}$ de casos & \% \\
\hline 2008 & 06 & 1,76 \\
2009 & 17 & 5,00 \\
2010 & 33 & 9,70 \\
2011 & 31 & 9,11 \\
2012 & 42 & 12,35 \\
2013 & 49 & 14,41 \\
2014 & 43 & 12,64 \\
2015 & 51 & 15,00 \\
2016 & 68 & 20,00 \\
TOTAL & $\mathbf{3 4 0}$ & $\mathbf{1 0 0}$ \\
\hline
\end{tabular}

Estes dados apresentam uma tendência crescente nos casos de $\mathrm{MC}$ em um período de 8 anos na cidade de Dourados-MS.

Tabela 2 - Tipos de Malformação Congênita registrada no município de Dourados/MS de 2008 a 2016.

\begin{tabular}{lcc}
\hline Tipo de Malformação & $\mathbf{N}^{\circ}$ de casos & \% \\
\hline Aparelho osteomuscular & 90 & 26,47 \\
Sistema Nervoso & 70 & 20,58 \\
Pé torto congênito & 45 & 13,23 \\
Aparelho digestivo & 23 & 6,76 \\
Aparelho geniturinário & 21 & 6,17
\end{tabular}




\begin{tabular}{lcc} 
& & Continuação \\
\hline Tipo de Malformação & $\mathbf{N}^{\circ}$ de casos & \% \\
\hline Fenda labial e fenda palatina & 17 & 5,00 \\
Aparelho circulatório & 14 & 4,11 \\
Espinha bífida & 09 & 2,64 \\
Anomalias cromossômicas NCOP* & 09 & 2,64 \\
Ausência atresia e estenose do intestino & 01 & 0,29 \\
delgado & & \\
Outras malformações congênitas & 41 & $\mathbf{1 0 0}$ \\
TOTAL & $\mathbf{3 4 0}$ &
\end{tabular}

* Não Classificado em Outra Parte

Conforme apresentado na Tabela 2, as maiores ocorrências de MC foram nos grupos de Recém-Nascidos com malformações do aparelho osteomuscular com 90 casos $(26,47 \%)$, seguidos pelo Sistema nervoso 70 casos $(20,58 \%)$, pé torto congênito $(13,23 \%)$, anomalias do sistema digestório 23 casos $(6,76 \%)$ e outras malformações não identificadas 41 casos $(12,05 \%)$.

Conforme apresentado na Tabela 3, no Mato Grosso do Sul foram 2.251 as ocorrências de MC notificadas no Estado no período de oito anos, sendo as do aparelho osteomuscular detentor do maior número de eventos, com 558 casos $(24,78 \%)$, seguido pelo pé torto congênito com $401(17,81 \%)$, sistema nervoso com $228(12,79 \%)$ e fenda labial e palatina com 183 casos $(8,12 \%)$.

Tabela 3 - Tipos de Malformações Congênita registrada no Estado do Mato Grosso do Sul de 2008 a 2016.

\begin{tabular}{lcr}
\hline Tipo de Malformação & $\mathbf{N}^{\circ}$ de casos & $\mathbf{\%}$ \\
\hline Aparelho osteomuscular & 558 & 24,78 \\
Pé congênito & 401 & 17,81 \\
Sistema Nervoso & 288 & 12,79 \\
Fenda labial e Palatina & 183 & 8,12 \\
Outras malformações congênitas & 254 & 11,28 \\
Aparelho geniturinário & 152 & 6,75 \\
Aparelho digestivo & 119 & 5,28 \\
Aparelho circulatório & 109 & 4,84 \\
Anomalias cromossômicas NCOP & 91 & 4,04 \\
Espinha bífida & 61 & 2,70 \\
Deformidades congênitas do quadril & 17 & 0,75 \\
Hemangioma e linfangioma & 09 & 0,39 \\
Testículo não-descido & 05 & 0,22 \\
Ausência atresia e estenose do intestino delgado & 04 & 0,17 \\
TOTAL & $\mathbf{2 . 2 5 1}$ & $\mathbf{1 0 0 , 0 0}$ \\
\hline
\end{tabular}

Já as ocorrências de MC notificadas a nível Nacional estão detalhadas na Tabela 4. No período de oito anos foram 202.410 casos, estando em primeiro o aparelho osteomuscular com 55.262 eventos $(27,30 \%)$ seguido pelo pé torto congênito com $25.256(12,47 \%)$, sistema nervoso $21.053(10,40 \%)$ e o aparelho circulatório 16.531 casos $(8,16 \%)$. 
Tabela 4 - Tipos de Malformações Congênitas registrada no Brasil de 2008 a 2016.

\begin{tabular}{lcr}
\hline Tipo De Malformação & $\mathbf{N}^{\mathbf{0}}$ de Casos & \multicolumn{1}{c}{$\%$} \\
\hline Aparelho osteomuscular & 55.262 & 27,3000 \\
Pé Torto congênito & 25.256 & 12,4700 \\
Sistema Nervoso & 21.053 & 10,4000 \\
Aparelho circulatório & 16.531 & 8,1600 \\
Outras malformações congênitas & 26.111 & 12,9000 \\
Aparelho geniturinário & 15.959 & 7,8800 \\
Fenda labial e fenda palatina & 13.553 & 6,6900 \\
Anomalias cromossômicas NCOP & 9.501 & 4,6100 \\
Aparelho digestivo & 8.565 & 4,2300 \\
Espinha bífida & 5.134 & 2,5300 \\
Testículo não-descido & 3.062 & 1,5100 \\
Deformidades congênitas do quadril & 1.093 & 0,5300 \\
Hemangioma e linfangioma & 897 & 0,4400 \\
Ausência atresia e estenose do intestino & 421 & 0,2000 \\
delgado & & \\
Outras afecções comp. teg. esp. RN & 08 & 0,0040 \\
Anomalias dentofaciais & 02 & 0,0009 \\
Sífilis congênita & 02 & 0,0009 \\
TOTAL & $\mathbf{2 0 2 . 4 1 0}$ & $\mathbf{1 0 0 , 0 0 0 0}$ \\
\hline
\end{tabular}

Percebe-se que as principais malformações que acometem os recém-nascidos têm semelhança do tipo de MC para as diferentes regiões. Sendo assim, foi correlacionado o tipo de malformação com o sexo/raça, idade materna, tipo de gestação, idade gestacional ao nascimento, número de consultas realizadas e via de parto.

Tabela 5 - Relação entre o número de consultas pré-natal, número de casos de malformações e percentual.

\begin{tabular}{lcr}
\hline $\mathbf{N}^{\mathbf{0}}$ de consultas & $\mathbf{N}^{\mathbf{0}}$ de casos & $\mathbf{\%}$ \\
\hline Nenhuma & 12 & 3,52 \\
1 a 3 consultas & 26 & 7,64 \\
4 a 6 consultas & 101 & 29,70 \\
7 ou mais & 198 & 58,23 \\
Ignorado & 03 & 0,88 \\
TOTAL & $\mathbf{3 4 0}$ & $\mathbf{1 0 0 , 0 0}$ \\
\hline
\end{tabular}

Dentre os nascidos vivos com malformações congênitas, a maior parte das mães realizaram 7 ou mais consultas $(58,23 \%)$, seguidas de mães que fizeram de 4 a 6 consultas de pré-natal $(29,70 \%)$ (Tabela 5$)$. 
Tabela 6 - Relação entre a idade gestacional, número de casos de malformações e percentual.

\begin{tabular}{lcr}
\hline Idade Gestacional & $\mathbf{N}^{\mathbf{0}}$ de casos & $\boldsymbol{\%}$ \\
\hline 22 a 27 semanas & 08 & 2,35 \\
28 a 31 semanas & 21 & 6,17 \\
32 a 36 semanas & 99 & 29,11 \\
37 a 41 semanas & 210 & 61,76 \\
42 semanas ou mais & 02 & 0,58 \\
\hline TOTAL & $\mathbf{3 4 0}$ & $\mathbf{1 0 0 , 0 0}$ \\
\hline
\end{tabular}

A maioria dos nascidos vivos com malformações congênitas nasceram a termo, ou seja, idade gestacional de 37 a 41 semanas, totalizando 210 nascimentos $(61,76 \%)$, acompanhado com 99 casos de bebês prematuros com idade gestacional entre 32 a 36 semanas $(29,11 \%)$ (Tabela 6).

Tabela 7 - Relação do tipo de gravidez, via de parto, número de casos de malformações e percentual.

\begin{tabular}{lcr}
\hline Gravidez & $\mathbf{N}^{\mathbf{0}}$ de casos & \% \\
\hline Única & 325 & 95,58 \\
Dupla & 12 & 3,52 \\
Tripla ou mais & 03 & 0,88 \\
Via de parto & & \\
Normal & 95 & 27,94 \\
Cesárea & 245 & 72,05 \\
TOTAL & $\mathbf{3 4 0}$ & $\mathbf{1 0 0 , 0 0}$ \\
\hline
\end{tabular}

Dos 340 nascimentos com MC a gestação única foi de 325 (95,58\%), posteriormente gemelares $12(3,52 \%)$ e tripla ou mais foi de 03 casos $(0,88 \%)$. A via de parto predominante foi a cesariana com 245 dos nascimentos com 72,05\% no município de Dourados e posteriormente parto normal 27,94\% (Tabela 7)

Tabela 8 - Relação entre a raça/cor, idade e instrução materna, número de casos de malformações e percentual.

\begin{tabular}{lcr}
\hline Raça/cor & $\mathbf{N}^{\mathbf{0}}$ de casos & \% \\
\hline Branca & 159 & 46,76 \\
Preta & 05 & 1,47 \\
Amarela & 01 & 0,29 \\
Parda & 130 & 38,23 \\
Indígena & 45 & 1,17 \\
Idade Materna & & \\
10 a 14 anos & 05 & 1,47 \\
15 a 19 anos & 60 & 17,64 \\
20 a 24 anos & 81 & 23,82 \\
25 a 29 anos & 78 & 22,94 \\
30 a 34 anos & 76 & 22.35 \\
35 a 39 anos & 34 & 10,00 \\
40 a 44 anos & 05 & 1,47 \\
45 a 49 anos & 0,1 & 0,29 \\
Instrução da Mãe & & \\
Nenhuma & 02 & 0,58
\end{tabular}


Vittalle - Revista de Ciências da Saúde v. 32, n. 2 (2020) 17-26

\begin{tabular}{lcr} 
& & Continuação \\
\hline Raça/cor & $\mathbf{N}^{\mathbf{0}}$ de casos & \% \\
\hline 1 a 3 anos & 14 & 4,11 \\
4 a 7 anos & 81 & 23,82 \\
8 a 11 anos & 164 & 48,23 \\
12 anos e mais & 77 & 22,64 \\
Ignorado & 02 & 0,58 \\
TOTAL & $\mathbf{3 4 0}$ & $\mathbf{1 0 0 , 0 0}$ \\
\hline
\end{tabular}

No que se refere à raça/cor encontrou-se que 46,76\% (159 casos) as malformações estão predominantes nas mulheres brancas, seguido de mulheres pardas 38,23\% (130 casos). Dentre as variáveis de idade materna verificou-se que a idade variou de 10 a 49 anos, sendo que a maioria foi de 20 a 24 anos com 81 mães $(23,82 \%)$. Quanto a instrução da mãe predominou de 8 a 11 anos de estudo 48,23\% (164 mães) e posteriormente 4 a 7 anos $23,82 \%$ (81 casos) (Tabela 8 ).

\section{Discussão}

As MC mais comuns entre os nascidos vivos na cidade de Dourados-MS foram do aparelho osteomuscular. Entre os nascidos vivos, predominaram as crianças cujas mães eram brancas, idade entre 20 a 24 anos de idade, com 8 a 11 anos de escolaridade, realizaram 7 ou mais consultas de pré-natal, tiveram seus filhos a termo, entre 37 a 41 semanas de gestação, via cesárea.

Como mostra a Tabela 3, os nascidos vivos no estado de Mato Grosso do Sul foram de 2.251 casos, sendo que a malformação que predominou foi do aparelho osteomuscular com 558 casos $(24,78 \%)$ e, posteriormente, quando levantados os casos de nível nacional, também houve predomínio do aparelho osteomuscular com 202.410 casos $(27,30 \%)$.

As consultas de pré-natal preconizada são de no mínimo seis, intercalando entre Médico e Enfermeiro. Além das consultas realizadas na unidade de saúde, a gestante poderá ser acompanhada também durante as visitas domiciliares. O número de consultas será programado de acordo com as condições clínicas da gestante, que determinam maior risco materno ou perinatal (5).

A média de notificações no estado de Mato Grosso do Sul está acima da nacional, conforme demonstrado nas tabelas 2 e 3, que evidenciam uma média de 7,13\% no âmbito estadual e de 5,87\% no âmbito federal.

$\mathrm{O}$ Estado tem como fonte de renda a agropecuária e, como mencionado anteriormente neste estudo, este é um fator que apresenta forte relação com o surgimento de casos de MC, devido ao uso de agrotóxico nas lavouras. Contudo, não podemos descartar os outros fatores de risco, como o uso de tabaco, bebida alcoólica, uso de medicamentos, entre outros (3-6).

Ao ser detectada alguma MC durante a gestação, a mesma será encaminhada para o serviço especializado e se torna Gestação de Alto Risco, ou seja, quando a mãe e/ou o bebê possuem riscos (5). Como vimos anteriormente na Tabela 5, 198 gestantes que tiveram seus filhos com malformações congênitas realizaram as consultas conforme preconizados pelo Ministério da Saúde.

Salienta Ricci (7) que a gestação dura em torno de 280 dias, sendo que é necessário subtrair os 14 dias que em média ocorre a fertilização do óvulo pelo espermatozoide em um ciclo menstrual de 28 dias, totalizando 266 dias de gestação ou 40 semanas a partir da data da última menstruação (DUM).

Reis e Ferrari (8) referem que a idade materna é um fator de risco para as MC, sendo 
assim, é sabido que mulheres abaixo de 20 anos ou acima dos 35 anos apresentam alto risco para certas malformações, como a Síndrome de Down, gastrosquise e outras disfunções vasculares.

Um estudo realizado com gestantes na faixa etária de 42 anos, concluiu que metade das gestações resultaram em aborto espontâneo, gravidez ectópica ou morte fetal intrauterina, e que esse resultado possivelmente transcorria do elevado número de conceptos impróprios à vida, devido a diminuição uterina e função hormonal (8).

Além disso, nesse mesmo estudo mostrou-se um elevado número de casos de malformações congênitas entre os recém-nascidos de mães com idade igual ou maior que 35 anos (8). No presente estudo, nota-se que a idade materna não foi parâmetro para maior incidência de malformações congênitas, pois as MC ocorreram tanto em genitoras acima de 19 anos como também antes dos 35 anos, inclusive em maior incidência, conforme evidenciado na Tabela 8 .

As anomalias tem como suas principais causas os transtornos congênitos e perinatais, em muitas ocasiões acompanhadas por agentes infecciosos nocivos durante $o$ desenvolvimento fetal, como por exemplo: vírus da rubéola, imunodeficiência humana (HIV), o vírus Zika, o citomegalovírus (CMV), treponema pallidum e o Toxoplasma gondii; além disso, destaca o uso de substancias lícitas e ilícitas, medicações teratogênicas e endocrinopatias maternas (6).

Contudo, algumas MC podem variar de acordo com os grupos raciais, como polidactilia pós-axial, muito comum em negros. A cardiopatia congênita, anencefalia, espinha bífida, fístula traquéo-esofágica e hipospádia na raça branca. Da mesma forma, em gestantes com fatores de risco como a diabetes mellitus, o bebê pode desenvolver MC do sistema nervoso central (SNC) e do aparelho cardiovascular. Hipertensão Arterial (HAS), Hipotiroidismo e Epilepsia estabelecem riscos moderados para Restrição de crescimento Intrauterino (CIUR) e microcefalia (8).

Um estudo realizado em 2017 envolvendo mães que foram expostas a solventes, pesticidas e metais pesados tinham maior risco em gerar filhos com fissura labial ou palato não sindrómico. Com isso o sexo masculino possui maior risco em desenvolver fissuras quando as mães eram expostas a solventes, enquanto o gênero feminino é mais propenso a desenvolver fissuras quando as mães estão expostas a poeira mineral (9).

Dentre as principais malformações levantadas o aparelho osteomuscular atinge o maior número de RN, uma vez que envolve o sistema ósseo e muscular, trabalham em conjunto para que ocorra o movimento, de forma que cada um desenvolva sua função. $\mathrm{O}$ sistema ósseo tem como função a proteção, sustentação, armazenamento de cálcio e potássio enquanto o sistema muscular permite a movimentação, sustentação entre outras funções (10).

As malformações que acometem o sistema nervoso estão relacionadas com a falha no fechamento do tubo neural que ocorre entre a $18^{\circ}$ e o $25^{\circ}$ semana de gestação. Sua etiologia é multifatorial e pode estar associada com causas genéticas, ambientais e desconhecidas, sendo considerada anomalias graves (11).

Melo et al (12) descreve que mães com idade inferior a 19 anos de idade possuem grandes chances de seus filhos nasceram com defeitos no tubo neural e baixo peso ao nascer (peso abaixo de $1.500 \mathrm{~g}$ ). Também ressalta que os fatores ambientais e genéticos podem ser os responsáveis pelas malformações nos recém-nascidos, e com isso implicar no baixo peso ao nascer.

O uso de ácido fólico por mulheres em idade fértil é fundamental, pois o mesmo é uma vitamina do complexo B e atua no desenvolvimento das células nervosas e na prevenção dos defeitos congênitos do tubo neural. É essencial para a síntese dos ácidos desoxirribonucleico (DNA) e ribonucleico (RNA), e é um dos elementos fundamentais 
na eritropoiese. A deficiência do mesmo está associada com defeitos do tubo neural no feto e na mãe e pode acarretar anemia megaloblástica. Quando suplementada ainda no início da gestação, pode reduzir em até $75 \%$ de chances do bebê nascer com malformações no tubo neural. Portanto, é preconizado que a suplementação de ácido fólico se inicie antes da concepção, com um tempo mínimo de trinta dias e durante a gestação pelo menos no primeiro trimestre (13).

A sífilis quando não tratada pode acarretar problemas para o bebê, a exemplo de anomalia óssea e atraso mental. A Rubéola acarreta problemas como a surdez, microcefalia, cegueira e cardiopatia. Toxoplasmose pode levar o desenvolvimento de hidrocefalia, cegueira, coriorretinite, atraso mental. Infecção por CMV alta exposição para restrição de crescimento intra-utero, atraso mental e surdez (8).

As gestações de fetos gemelares apresentam maiores chances de desenvolver malformações congênitas quando comparadas com gestações de fetos únicos, e justifica que a gestação por si só pode apresentar algum tipo de problema, pois os fetos estão em desenvolvimento no mesmo período. No presente estudo, o número de casos gemelares não foi relevante para constatar a informação trazida pelo autor (14).

Os casos de MC são maiores em gestações múltiplas, principalmente em gestações monozigóticas, quando comparadas com gestações únicas. O mesmo autor descreve que, nos gemelares que tem alguma malformação, os casos de aborto espontâneo são três vezes maiores comparadas as gestações únicas (15).

As MC podem ser reduzidas por meio da educação em saúde. Orientações com a população alvo - ou seja, mulheres em idade fértil - sobre importância da realização das consultas de pré-natal, calendário vacinal completo, o não prejuízo da gestação pelas rotinas diárias e laborais, o uso cauteloso de medicamentos, e não consumir as bebidas alcóolicas, cigarro e drogas ilícitas (16).

\section{Conclusão}

Ao analisar as MC notificadas na cidade de Dourados no período de nove anos (20082016), os números se apresentaram de forma crescentes. Em um comparativo realizado entre os níveis estadual e nacional, observa-se que os casos levantados de MC são análogos. Concluiu-se que, no estado de Mato Grosso do Sul, houve um aumento nos casos notificados, onde existem vários fatores de risco que podem levar às malformações, como o uso de agrotóxicos, fatores hereditários, aspectos nutricionais, uso de medicações teratogênicas ou até mesmo a não suplementação de ácido fólico como recomendado. Além disso, segundo informações respaldadas na literatura pesquisada, salienta-se a importância da suplementação de ácido fólico, o uso cauteloso de medicamentos, o não consumo de bebidas alcóolicas, cigarros e drogas ilícitas. As consultas de pré-natal foram realizadas como preconizado pelo ministério da saúde, sendo assim, conclui-se que as gestantes realizaram sete ou mais consultas. Quanto a idade gestacional, os bebês nasceram a termo, de gestações únicas, via cesariana. A idade materna variou de 10 a 49 anos, sendo que a maioria foi de 20 a 24 anos. Predominou a raça/cor branca e mães com instruções de 8 a 11 anos de escolaridade. A média de notificações no estado de Mato Grosso do Sul está acima da nacional, com cerca de $7,13 \%$, enquanto que no âmbito federal encontra-se uma média de 5,87\%. Conclui-se que as malformações estão aumentando no decorrer dos anos, e para que haja uma redução é necessário realizar educação em saúde com mulheres, principalmente as que estão em período fértil na idade de 20 a 34 anos, sobre importância da realização das consultas de pré-natal. 


\section{Referências}

1. World Health Organization. Congenital anomalies. Fact sheet $\mathrm{n}^{\circ}$ 370. Updated January 2014. Disponível em: http://www.who.int/mediacentre/factsheets/fs370/en/. Acessado em: 19 de julho de 2020.

2. BRASIL. Ministério da Saúde. Departamento de Informações do Sistema Único de SaúdeDATASUS, 2017.

3. Oliveira NP, Moi GP, Santos MA, Silva AMC, Pignati WA. Malformações Congênitas em municípios de grande utilização de agrotóxicos em Mato Grosso, Brasil. Revista Ciência \& Saúde 2014; 19(10): 4123- 4130.

4. Dutra LS, Ferreira AP. Associação entre malformações congênitas e a utilização de agrotóxicos em monoculturas no Paraná, Brasil. Revista Saúde e Debate 2017; 41: 241-253.

5. BRASIL, Ministério da Saúde. Manual Técnico de Gestação de baixo risco. 5 ed. BRASILIA: MINISTÉRIO DA SAÚDE, 2012; p 302.

6. Mendes IC, Jesuino RSA, Pinheiros DS, Rebelo ACS. Anomalias congênitas e suas principais causas evitáveis: uma revisão. Revista Médica de Minas Gerais 2018; 28: e-1977.

7. Ricci, S. S. Enfermagem Materno-neonatal e Saúde da Mulher. $3^{\circ}$ ed. Rio de Janeiro: Guanabara Koogan, 2015.

8. Reis LLAS, Ferrari R. Malformações congênitas: perfil sociodemográfico das mães e condições de gestação. Revista de Enfermagem UFPE online 2014; 8(1): 98-106.

9. Azevedo PB, Rocha AG, Keim LMN, Lavinsky D, Furtado GV, Mattos EP, Vargas FR, Leotti VB, Saraiva PML, Jardim LB. Ophthalmological and Neurologic Manifestations in Pre-clinical and Clinical Phases of Spinocerebellar Ataxia Type 7. The Cerebellum 2019; 18(3): 388-396.

10. Luz GS, Karam SM, Dumith SC. Anomalias congênitas no estado do Rio Grande do Sul: análise de série temporal. Revista Brasileira de Epidemiologia 2019; 22(29): 01-14.

11. Pereira ALA, Souza MAB, Santos JC. Tendência Temporal das malformações congênitas do sistema nervoso nos últimos quatros anos do Brasil. Revista Pesquisa de Fisioterapia 2018; 8(1): 16- 23.

12. Melo, WA, Zurita, RCM, Uchimura TT, Marcon SS. Anomalias congênitas: fatores associados à idade materna em município sul brasileiro, 2000 a 2007. Revista Eletrônica de Enfermagem 2010; 12(1): 73-82.

13. Linhares AO, Cesar JA. Suplementação com ácido fólico entre gestantes no extremo Sul do Brasil: prevalência e fatores associados. Revista Ciências \& Saúde Coletiva 2017; 22(2): 535- 542.

14. Brito APM, Ribeiro KRA, Duarte VGP, Abreu EP. Enfermagem no contexto familiar na prevenção de anomalias congênitas: revisão integrativa. Journal of Health \& Biological Siences 2019; 7(1): 64-74.

15. Rodrigues LS, Lima RHS, Costa RF, Batista RFL. Características das crianças nascidas com malformações congênitas no município de São Luís, Maranhão, 2002-2011. Revista em epidemiologia em Serviço e saúde 2014; 23(2): 295-304.

16. Santos RS, Dias IMV. Refletindo sobre a malformação congênita. Revista Brasileira de Enfermagem 2005; 58(5): 592-596. 